\begin{abstract}
Iranica
Abstracta Iranica Revue bibliographique pour le domaine irano-aryen

Volume 37-38-39 | 2018

Comptes rendus des publications de 2014-2016

\section{Kamal Aldin Niknami, Sona Naderi,. « Sasanian Clay Sealing Collection in the Bandar Abbas Museum of Iran »}

Rika Gyselen

\author{
(2) OpenEdition \\ Journals \\ Édition électronique \\ URL : http://journals.openedition.org/abstractairanica/42627 \\ DOI : 10.4000/abstractairanica.42627 \\ ISBN : 1961-960X \\ ISSN : 1961-960X \\ Éditeur : \\ CNRS (UMR 7528 Mondes iraniens et indiens), Éditions de l'IFRI
}

\title{
Référence électronique
}

Rika Gyselen, « Kamal Aldin Niknami, Sona Naderi,. « Sasanian Clay Sealing Collection in the Bandar Abbas Museum of Iran » », Abstracta Iranica [En ligne], Volume 37-38-39 | 2018, document 7, mis en ligne le 10 mars 2018, consulté le 28 septembre 2020. URL : http://journals.openedition.org/ abstractairanica/42627 ; DOI : https://doi.org/10.4000/abstractairanica.42627

Ce document a été généré automatiquement le 28 septembre 2020.

Tous droits réservés 


\title{
Kamal Aldin Niknami, Sona Naderi,. «Sasanian Clay Sealing Collection in the Bandar Abbas Museum of Iran »
}

\author{
Rika Gyselen
}

\section{RÉFÉRENCE}

Kamal Aldin Niknami, Sona Naderi,. « Sasanian Clay Sealing Collection in the Bandar Abbas Museum of Iran », ANES, 53, 2016, p. 219-241.

1 Il ne s'agit évidemment pas d'un 'hoard' ni d'une 'archive' comme le disent les auteurs - l'argument avancé en faveur d'une telle interprétation (p. 229) est bien fragile mais d'une composition aléatoire de quelques 800 bulles qui circulaient dans le commerce des antiquités et qui, saisies, ont été intégrées dans les collections du Musée de Bandar Abbas. L'objectif de la contribution est de présenter une classification et une description des principales bulles d'argile de la collection, bien qu'il reste quelque 700 autres à publier! On peut espérer que les auteurs opteront pour une publication électronique ce qui permettra d'avoir des photos lisibles, un problème récurrent lorsqu'on publie des bulles.

2 L'article est composé d'une introduction générale sur la fonction et les types de bulles suivie d'un catalogue d'une petite sélection de bulles. La présentation générale des bulles de la collection est assortie d'illustrations graphiques de quelques formes de bulles. Certaines remarques devraient être plus nuancées, en particulier celles propageant l'idée que les bulles sont nécessairement cuites pour pouvoir être conservées. Cela impliquerait-il que les bulles encore attachées à des documents en parchemin ou textile ont aussi été cuites?

3 Suit la présentation des bulles administratives au nombre de cinq dont les quatre sceaux administratifs sont connus (le renvoi à la publication a été oublié pour le sceau I/4: Gignoux / Gyselen, Studia Iranica Cahier 4, 1987; Gyselen 1993, Catalogue des 
sceaux... de la Bibliothèque Nationale de France) et une présentation des principaux motifs sur les bulles (p. 222-228). D'après le diagramme illustrant la fréquence des motifs dans la collection (p. 226, fig. 3) symboles et monogrammes forment la majorité. Bien que la bibliographie de cet article soit récente, il manque la référence vers un essai de lecture des monogrammes (R. Gyselen / Y. Monsef, « Décryptage de noms propres sur des monogrammes sassanides (1)», R. Gyselen (ed.), Objets et documents inscrits en pārsīg [Res Orientales, XXI], 2012, p. 149-178), alors que d'autres articles dans le même volume (ResOr XXI) sont mentionnés. Quelques remarques ont été consacrées à 'l'archive' et l'utilisation des sceaux dans le cadre légal (p. 224-225). On peut hésiter à suivre certains points de vue des auteurs, ainsi «It seems probable that private seals which stated the name of their owners in an inscription were intended to signify the owner's wealth and status, rather than to read for identification purposes ».

La conclusion aborde plusieurs aspects concernant les sceaux administratifs dont certains sont un peu hors propos par rapport à cette collection.

5 Le catalogue (p. 231-238) est présenté de façon claire: une photo de l'avers (I/3 est inversée et plusieurs sont à peine lisible), une description de la bulle et des différentes empreintes. Il aurait été utile d'ajouter la position des divers sceaux : une donnée qui permet souvent de distinguer des bulles portant les mêmes empreintes. Bizarrement aucune lecture des sceaux administratifs n'est donnée ici - mais elle figure p. 223, fig. 2. Le souci d'économie éditoriale est probablement à l'origine de la réduction photographique des bulles. Ces objets ne sont déjà pas très faciles à lire lorsqu'ils sont reproduits à l'échelle 1:1, mais réduits cela devient souvent impossible. Dans les plus récentes publications de bulles, la norme est de publier les photographies des bulles à l'échelle 1:1, puis de reproduire chaque empreinte à l'échelle 2:1. Il faut espérer que la publication de la collection complète suivra cette norme. Il est en effet bien frustrant de ne pas pouvoir bien distinguer les motifs (et les inscriptions) alors que beaucoup de motifs sont bien intéressants. Quelques oublis de lectures d'inscriptions sont à noter. Beaucoup d'empreintes sont identiques à celles des bulles de la collection Saeedi. L'auteur ne mentionne le parallèle que dans un seul cas. On peut apprécier que les auteurs aient scrupuleusement suivi le système de translittération et transcription de référence (MacKenzie 1972).

\section{AUTEURS}

\section{RIKA GYSELEN}

CNRS, Mondes iranien et indien 